\title{
Structure of Dengue Virus: Implications for Flavivirus Organization, Maturation, and Fusion
}

\author{
Richard J. Kuhn ${ }^{1,3}$, Wei Zhang ${ }^{1}$, Michael G. Rossmann ${ }^{1}$, Sergei V. Pletnev ${ }^{1}$, Jeroen \\ Corver $^{2}$, Edith Lenches ${ }^{2}$, Christopher T. Jones ${ }^{1}$, Suchetana Mukhopadhyay ${ }^{1}$, Paul R. \\ Chipman $^{1}$, Ellen G. Strauss ${ }^{2}$, Timothy S. Baker ${ }^{1}$, and James H. Strauss ${ }^{2}$ \\ ${ }^{1}$ Department of Biological Sciences, Purdue University, West Lafayette, Indiana 47907 \\ ${ }^{2}$ Division of Biology 156-29, California Institute of Technology, Pasadena, California 91125
}

\section{Summary}

The first structure of a flavivirus has been determined by using a comThe first structure of a flavivirus has been determined by using a combination of cryoelectron microscopy and fitting of the known structure of glycoprotein $\mathrm{E}$ into the electron density map. The virus core, within a lipid bilayer, has a less-ordered structure than the external, icosahedral scaffold of 90 glycoprotein $\mathrm{E}$ dimers. The three $\mathrm{E}$ monomers per icosahedral asymmetric unit do not have quasiequivalent symmetric environments. Difference maps indicate the location of the small membrane protein $\mathrm{M}$ relative to the overlaying scaffold of $E$ dimers. The structure suggests that flaviviruses, and by analogy also alphaviruses, employ a fusion mechanism in which the distal $\beta$ barrels of domain II of the glycoprotein $\mathrm{E}$ are inserted into the cellular membrane.

\section{Introduction}

The flaviviruses (Latin flavus meaning yellow, because of the jaundice induced by yellow fever virus) comprise a large genus of medically important, arthropod-transmitted, enveloped viruses with members that include yellow fever, dengue, West Nile, tick-borne encephalitis (TBEV), and Japanese encephalitis viruses (Burke and Monath, 2001; Lindenbach and Rice, 2001). Dengue virus is one of the most significant human viral pathogens transmitted by mosquitoes and causes 50 million or more cases of infection worldwide each year, resulting in around 24,000 deaths (World Health Organization, 1998). Infection is usually characterized by fever and severe joint pain, but more serious syndromes, dengue hemorrhagic fever or dengue shock syndrome, occur sometimes following dengue infection. Dengue hemorrhagic fever was mostly confined to Southeast Asia until the 1960's, when it also became endemic in Central America and, more recently, in South America. There are four distinct serotypes of dengue virus, and it has been postulated that hemorrhagic fever, or shock syndrome, is usually the result of sequential infection with multiple serotypes. Although vaccines have been developed for several flaviviruses, control of dengue virus through the use of vaccination has proved to be elusive (Burke and Monath, 2001). 
Electron micrographs showed that dengue virions are characterized by a relatively smooth surface, with a diameter of approximately $500 \AA$, and an electron-dense core surrounded by a lipid bilayer. In addition to the plus-sense RNA genome of $\sim 10,700$ nucleotides, there are three structural proteins that occur in stoichiometric amounts in the particle: core (C, 100 amino acids), membrane (M, 75 amino acids), and envelope (E, 495 amino acids). The atomic structure for the homologous E protein of TBEV has an elongated shape consisting of a central domain (I) that connects an Ig-like domain (III) to a dimerization domain (II) (Rey et al., 1995). Based on the shape of the molecule and the location of antibody epitopes, Rey et al. (1995) postulated that the E protein would lie flat along the surface of the virus lipid bilayer.

Alphaviruses (including Sindbis, Semliki Forest, and Ross River viruses) and flaviviruses were formerly considered genera within the togavirus family because of their similar structural organization. However, they differ in gene order and replication strategy and are now classified into separate families (Strauss and Strauss, 2001). In contrast to the smooth surface of the flaviviruses, the alphaviruses have prominent spikes that project from the bilayer membrane (Cheng et al., 1995). Despite these differences, recent studies (Lescar et al., 2001; Pletnev et al., 2001) have shown that there is a close structural and functional relationship between the flavivirus and alphavirus E and E1 glycoproteins, respectively, demonstrating that they both form icosahedral scaffolds. These glycoproteins participate in the process of membrane fusion (Kielian et al., 1996; Allison et al., 2001).

We report the structure of dengue virus type $2 \mathrm{~S} 1$ strain, a vaccine candidate derived from the PR-159 isolate (Hahn et al., 1988). A three-dimensional image reconstruction shows that the virion has a well-organized outer protein shell, a lipid bilayer membrane, and a lesswell-defined inner nucleocapsid core. The known atomic structure of the homologous $\mathrm{E}$ protein dimer of TBEV (Rey et al., 1995) has been fitted into the outer layer of density in the cryoelectron microscopy (cryoEM) reconstruction. The icosahedral scaffold consists of 90 such dimers with three monomers in the icosahedral asymmetric unit but lacking $T=3$ quasiequivalent environments (Caspar and Klug, 1962). The glycoprotein organization suggests a common, class II fusion mechanism for alpha- and flaviviruses based on the insertion of a $\beta$ barrel-type structure into the host cell membrane. Class I and II fusion proteins were first differentiated by Lescar et al. (2001) based on a description of a variety of properties related to the activation and position of the fusogenic peptide. Here we have emphasized the structural and mechanistic differences between these fusion classes.

\section{Results and Discussion}

\section{The Virus Organization}

The $24 \AA$ resolution cryoEM reconstruction of dengue virus particles (Figure 1A) assumed icosahedral symmetry. This was readily justified by the convergence of stable values for angles defining particle orientations, acceptable correlation coefficients, and the good agreement between reconstructions of two independent data sets to at least $24 \AA$ A resolution.

The external, $250 \AA$ A radius of dengue virus was found to be consistent with earlier measurements of flaviviruses (Burke and Monath, 2001; Lindenbach and Rice, 2001). Size 
comparisons with other viruses, along with knowledge of the mass of the E glycoprotein, suggested that dengue virus might have three (Ferlenghi et al., 2001) or possibly four (as is the case for alphaviruses [Paredes et al., 1993; Cheng et al., 1995]) subunits of E per icosahedral asymmetric unit. A radially averaged density distribution (Figure 1B) of the cryoEM reconstruction shows that the density can be divided into a series of spherical shells. The highest density occurs in the outermost shell between radii of 220 and $245 \AA$, and this density is consistent with the known atomic structure of TBEV E glycoprotein (see below). The next shell down, between radii 185 and $220 \AA$, has density that is only about $60 \%$ in height of the outer shell, but nevertheless it has clearly defined features. These features represent the ectodomains of the M proteins (38 amino acids) and the stem regions of the Eglycoproteins (52 amino acids).

Immediately inside these external protein shells, between radii of 140 and $185 \AA$, are two concentric layers of electron density, which are consistent with the density distribution expected for a lipid bilayer. Not only is the $45 \AA$ thickness similar to other measurements of lipid bilayers, but also the inner and outer leaflets of higher, featureless density, representing the phosphate head groups, are separated by a shell of very low density. Furthermore, these results are within the experimental error of the observed lipid content and buoyant densities for flaviviruses (see Experimental Procedures).

Internal to the putative lipid region is the nucleocapsid shell situated between radii of 105 and $135 \AA$ A. The density in the nucleocapsid shell has distinct globular features representing the core protein subunits (Figure 2A). However, unlike the alphaviruses, which have a distinct nucleocapsid (Paredes et al., 1993; Cheng et al., 1995), the maximum height of the density of the dengue nucleocapsid shell is only $50 \%$ of that of the external glycoprotein. This suggests that the core protein is poorly ordered or that the orientation of the core as a whole is somewhat variable relative to the external glycoprotein scaffold. In alphaviruses, the core obtains its icosahedral symmetry from the external glycoprotein scaffold while budding from the cell (Forsell et al., 2000; Pletnev et al., 2001) and forming specific contacts with the carboxy termini of the E2 glycoproteins (Lee et al., 1996; Skoging et al., 1996). Such a specific interaction has not been demonstrated for flaviviruses. Although the size of the nucleocapsid is substantially smaller than the $150 \AA$ radius observed for many $T$ $=3$ virus capsids (Baker et al., 1999), the protein shell is significantly thinner than the usual $\sim 45 \AA$ seen, for instance, in picornaviruses. This is, in part, the consequence of the molecular weight of the dengue virus core protein being only one-third of the molecular weight of the picornavirus capsid protein. The cage-like structure of the core and its reduced order provide enough space for the RNA genome, which may extend to the outer radius of the core (see Experimental Procedures and Figure 2). Notwithstanding the possible lower icosahedral order of the nucleocapsid shell, the density within the core shell has recognizable features, showing extended $20 \AA$ thick, low-density rods that are reminiscent of icosahedrally ordered RNA (Figure 2B; Chen et al., 1989; Larson et al., 1993).

\section{Interpretation of the CryoEM Map Using the Known Structure of the E Glycoprotein}

Trypsin treatment of TBEV yields a dimerized fragment of E that lacks the last 101 amino acids and whose X-ray crystallographic structure has been determined (Rey et al., 1995). In 
the absence of any guidance from quasi-symmetry (Figures 1A and 3A), a quantitative technique was used for comparing different fits of the $\mathrm{E}$ glycoprotein dimer X-ray structure into the dengue cryoEM map by using the EMfit program (Rossmann, 2000; see Experimental Procedures). The procedure consisted of fitting the dimers, one at a time, until essentially all the higher density features were occupied. The first set of dimers was found to be on the icosahedral 2-fold axes (Table 1), whereas the second set was positioned on quasi-2-fold axes (Table 2). Thus, three monomers were placed into an icosahedral asymmetric unit.

The overall fit of the 90 TBEV E dimer structures to the cryoEM map of dengue virus gives a satisfying, close-packing arrangement (Figures 3B and 3D). The dengue virus structure (Figure 3C) has sets of three, nearly parallel dimers. These sets associate to form a "herringbone" configuration on the viral surface. This arrangement markedly differs from a true $T=3$ structure because the dimers on the icosahedral 2-fold axes do not have a quasi-3fold relationship to the dimers on the quasi-2-fold axes. The environment of the dimers on the icosahedral 2-fold axes is totally different from the environment of the dimers on the quasi-2-fold axes. This could have significant consequences for nonsymmetric binding of cellular receptors and antibodies. The three nearly parallel dimers form a dominant association with each other that supersedes the interactions between individual monomers in the assembled icosahedral particle (Figure 3C). Should the close association between the three dimers within each set be weakened (for instance, by lowering the $\mathrm{pH}$ ), then there would be a tendency to produce a $T=3$ structure in which every monomer has an equivalent environment.

It was proposed by Rey et al. that the slight curvature of the TBEV E dimer corresponds to the curvature of the roughly spherical virion (Rey et al., 1995). This conclusion is supported by extensive studies of antibody binding sites (Mandl et al., 1989; Roehrig et al., 1998) and heparin sulfate binding sites on the viral surface (Mandl et al., 2001). The present structure of mature dengue virus is consistent with these observations.

Although the E1 glycoprotein of the alphaviruses has a similar fold as the E protein of TBEV and performs a similar function, the organization of dimers is dissimilar (Lescar et al., 2001; Pletnev et al., 2001). Whereas in alphaviruses monomers are associated "back-toback" within a dimer with their long directions making an angle of about $40^{\circ}$ with each other, in flaviviruses the monomers are "face-to-face" within a dimer with their long directions being essentially antiparallel to each other. Although the tertiary structural similarity points to a common evolutionary origin of the glycoprotein genes in these two virus families, their functional deployment is quite different.

The atomic structure obtained by fitting the TBEV E dimers into the cryoEM dengue virus map was used to calculate a $24 \AA$ resolution map. After appropriate scaling (Pletnev et al., 2001), this calculated map was subtracted from the cryoEM dengue virus density distribution. There were only three significant peaks in the difference map external to the lipid membrane, with heights of $\sim 0.6$ of the largest density in the original cryoEM map. They were positioned at structurally equivalent sites, below the prominent holes in the fitted dimers (Figures 1C, 1D, and 3), corresponding to the light blue density in Figure 1B. 
Possibly, these peaks represent the sites of the M proteins. Since the precursor membrane protein (prM) can mask or enhance monoclonal antibody binding sites on the external surface of E (Rey et al., 1995), the prM polypeptide would bind to the exterior of the $E$ protein, protecting the fusion peptide, which is in close proximity to the holes (Figure 3C), until prM is cleaved (Heinz et al., 1994).

The difference map (see above) also showed other, slightly lower, uninterpreted, extended density surrounding the putative $M$ protein peaks. The positions of these densities suggest that they may represent the 52 stem residues of the $\mathrm{E}$ glycoprotein between the trypsin cleavage site and the transmembrane region. The $\mathrm{C}$ termini of the $\mathrm{M}$ and $\mathrm{E}$ proteins may heterodimerize (Allison et al., 1999) above the membrane in a manner similar to the association of the E1 and E2 glycoproteins in alphaviruses (Heinz et al., 1994; Lescar et al., 2001; Pletnev et al., 2001; E.G.S., E.L., and J.H.S., unpublished results).

\section{Comparison with Recombinant Subviral Particles}

Coexpression of flavivirus $\mathrm{E}$ and prM in cell culture results in the self-assembly of small recombinant subviral particles (RSP) possessing an external shell of $\mathrm{E}$ and $\mathrm{M}$ with an internal membrane (Allison et al., 1995b). These particles appear to be immunologically identical to infectious virions and similar to small particles that are frequently produced in flavivirus-infected cells (Russell et al., 1980). The RSPs were shown to be fully competent in membrane fusion assays displaying kinetics similar to native virions (Corver et al., 2000). CryoEM and image reconstruction of RSPs by Ferlenghi et al. (2001) showed these to have $T=1$ icosahedral symmetry with TBEV E dimers located on icosahedral 2-fold axes. The location of these dimers is almost identical to the dimers that are found on the 2-fold axes of the dengue reconstruction. However, their extrapolation of the $T=1$ structure to larger particles with $T=3$ symmetry produced a virion model radically different from the flavivirus structure described here. Although it now transpires that their proposed $T=3$ structure is not that seen for the mature dengue virus, it is a seductively attractive model that may be structurally related to the fusogenic, low-pH form of the virus because it has obvious trimeric units, consistent with biochemical data (Heinz and Allison, 2000), and because, unlike mature virus, it exposes viral membrane. If this $T=3$ model for the fusogenic state of the virus is correct, then the dimers seen in the mature virus would have to undergo large rotations in the transition to the fusogenic form. During these rearrangements, it would be necessary for the virus to expand radially by at least $10 \%$ to avoid steric clashes (Figure 4).

\section{Fusion}

By far, the most studied virus-cell fusion process is for orthomyxoviruses. Elegant crystallographic studies have shown that fusion is initiated by the formation of a trimeric coiled-coil helix adjacent to the fusion peptide on the virus exterior, the insertion of this fusion peptide into the host cell membrane, and the subsequent formation of a 6 helix bundle (Skehel and Wiley, 1998). Flavi-(Heinz and Allison, 2000) and alphaviruses (Kielian, 1995) each possess a hydrophobic sequence of about a dozen amino acid residues known to be essential for fusion. These sequences are components of $\beta$ barrel-type structures (Rey et al., 1995; Lescar et al., 2001). Two kinds of protein structures occur in membranes: helices (associated with class I fusion), first discovered in bacterial rhodopsin (Subramaniam and 
Henderson, 2000), and $\beta$ barrels (proposed here for class II fusion) in porin structures (Schulz, 2000). Both helices and $\beta$-barrels hide all main chain amino and carbonyl polar groups by forming hydrogen bonding networks. The exteriors of these structures are hydrophobic to help accommodate them among the aliphatic chains that constitute a membrane. The similarities between membrane insertion of the alphavirus fusion peptide and insertion of $\beta$ barrel pore-forming toxins have been previously mentioned by analogy with the cholesterol-dependent cytolysins (Vashishtha et al., 1998).

The fusion peptides of flavi- and alphaviruses (Kielian, 1995; Allison et al., 2001) are located in the homologous E (Rey et al., 1995) and E1 (Lescar et al., 2001) glycoproteins, respectively. Furthermore, although the hydrophobic fusion peptides are not in equivalent positions, they are in neighboring loops of the $\beta$ structure at the distal end of domain II. Changes in the antigenic properties of the flaviviruses suggest that this domain makes the largest structural changes (Rey et al., 1995) when the $\mathrm{pH}$ is lowered, as is required for the fusion process to be initiated. The neutral $\mathrm{pH}$ crystallographic structures show that the tip of domain II for both flavi-and alphaviruses consists of a set of three antiparallel $\beta$ ribbons that do not form a closed $\beta$ barrel, as in a porin structure and as might be expected for a structure required to be able to insert itself into a membrane (Figure 5). Thus, we hypothesize that the conformation of the distal end of domain II changes when the $\mathrm{pH}$ is lowered to complete the hydrogen bonding network and to create a closed $\beta$ barrel.

The conformational changes (Allison et al., 1995a) required for fusion would have to include not only the closure of the $\beta$ barrel, but also the projection of these $\beta$ barrels toward the cellular membrane and the exposure of a significant area of viral membrane between the fusogenic anchors. For alphaviruses, this would require the displacement of the protecting E2 glycoprotein spikes, which would expose an area of viral membrane within the E1 trimer (Figure 5A). In dengue virus, the $\mathrm{E}$ dimers are so closely packed that the viral membrane remains inaccessible and fusion is impossible at neutral $\mathrm{pH}$. We, therefore, hypothesize that fusion is initiated by the low $\mathrm{pH}$-induced conformational change, creating the predicted $T=$ 3 structure (see above). This would expose surface area of the viral membrane (Figure 5B), requiring particle expansion.

The predicted conformational changes for class II fusion result in a process that is much faster than that observed for class I fusion (Corver et al., 2000), probably the result of synchronous motion provided by the icosahedral scaffold and the close association of the class II fusion proteins to one another. To initiate fusion, the lack of organization in class I envelopes requires the release of the fusion peptide and the interaction of multiple subunits to form a fusion pore. Since these subunits are not organized in an ordered array on the virus, time is required for the subunits to oligomerize and form the active fusion pore. In contrast, the icosahedral scaffold and the tight interactions between the envelope proteins in class II viruses provide a situation in which the fusion pores are already well ordered. 


\section{Experimental Procedures}

\section{Sample Preparation}

Dengue-2 virus, strain PR159-S1 (Hahn et al., 1988), was grown in mosquito C6/36 cells adapted to Eagle's minimum essential medium. Cells were infected at low multiplicity $(\sim 0.1)$ and maintained at $30^{\circ} \mathrm{C}$. After $48 \mathrm{hr}$, the medium was removed, the cells were reseeded using fresh medium, and the virus was harvested $72 \mathrm{hr}$ later. Following clarification, the virus was precipitated with $8 \%$ polyethylene glycol 8000 , and the resuspended virus was purified by sedimentation for $1 \mathrm{hr}$ at $32,000 \mathrm{rpm}$ and $4{ }^{\circ} \mathrm{C}$ in potassium tartrate step gradients (10\%-40\% potassium tartrate, $7.5 \%-30 \%$ glycerol). The visible band of virus was removed with a pipette and concentrated using Millipore Centricon centrifuge filters. The resulting virus preparation, in $50 \mathrm{jxl}$, had a titer of $2 \times 10^{10} \mathrm{pfu} / \mathrm{ml}$, which represented a recovery of $50 \%$ of the starting infectivity. It was dialyzed against 50 $\mathrm{mM}$ Tris (pH 7.6), $75 \mathrm{mM} \mathrm{NaCl}, 1 \mathrm{mM}$ EDTA, using microdialysis discs. Although preparations of dengue-2 from mosquito cells are often reported to contain large amounts of uncleaved prM, our preparations contained little or no prM as determined by gel electrophoresis followed by staining with Coomassie Brilliant Blue (data not shown). The ice-embedded dengue-2 virus sample was recorded in a Philips CM200 field emission gun transmission electron microscope under low-dose condition $\left(25.5 \mathrm{e}^{-} / \AA^{2}\right)$ at a magnification of 50,000. Twenty-five micrographs taken at defocus levels between 0.79 and $1.92 \mu \mathrm{m}$ were used to select 526 particles for the reconstruction. The experimental data were corrected with the phase contrast transfer function. The common line method was used to generate the initial model, and the model-based polar-Fourier transform method was used in computing the orientation for each viral image. The three-dimensional reconstruction was calculated by weighting each image by a function based on the phase contrast function and real-space correlation coefficient. The average real-space correlation coefficients (for a description, see Baker et al. [1999]) for all particles, compared in a 140-258 A radius range, was 0.43 with a standard deviation of 0.117 .

\section{Lipid Composition}

Alphaviruses have a molecular weight of about $52 \mathrm{MDa}$, calculated from their known composition (240 copies each of E1, E2, and C of known sequence, one copy of genomic RNA of $11.7 \mathrm{kB}$, and 30\% lipid; see Strauss and Strauss, 1994). Of this, about $15 \mathrm{MDa}$ is lipid, calculated from the reported 30\% lipid content of virions (reviewed in Lenard, 1980). Flavivirus virions have a molecular weight of about $22 \mathrm{MDa}$, calculated from their content of 180 copies of $\mathrm{E}, \mathrm{M}$, and $\mathrm{C}$ (this paper) of known sequence (reviewed in Chambers et al., 1990), one copy of genomic RNA of $10.9 \mathrm{kB}$, and a lipid content of $17 \%$ (reviewed in Russell et al., 1980). Estimates of the lipid content of flaviviruses have varied widely because of the difficulties of preparing suitable amounts of purified flavivirions for analysis, and more modern analyses would be useful. If the lipid content is $17 \%$, which appears to be the most reliable estimate reported in the literature, the flavivirus virion would contain 3.7 MDa of lipid, about one-quarter of the amount present in alphaviruses.

Alphaviruses have a lipid bilayer about $46 \AA$ thick centered at $R=232 \AA$. Thus, a volume of $31 \times 10^{6} \AA^{3}$ is available for the alphavirus lipids. We have shown here that flaviviruses have 
a lipid bilayer centered at $R=163 \AA$ and a thickness of $\sim 45 \AA$. Thus, the volume available for the flavivirus lipids is $16 \times 10^{6} \AA^{3}$. Hence, flaviviruses have a bilayer volume that is half the alphavirus volume, but they appear to contain only one-fourth as much lipid. Therefore, (1) the flavivirus lipids are packed less densely, perhaps because of the more extreme curvature of the bilayer, (2) the lipid composition is higher and flaviviruses contain the same percentage of lipid as alphaviruses, or (3) the lipid bilayer is centered below $163 \AA$, which we consider unlikely. In any event, it is very unlikely that the bilayer is centered above $R=$ $163 \AA$.

Alphaviruses, containing $30 \%$ lipid, have a calculated density of about $1.18 \mathrm{gm} / \mathrm{cm}^{3}$, based solely upon their composition and the densities of the separate components, close to the observed value of $1.21-1.22 \mathrm{gm} / \mathrm{cm}^{3}$ obtained by centrifugation in sucrose solutions (reviewed in Strauss and Strauss, 1994). Flaviviruses containing 17\% lipid have a calculated density of $1.3 \mathrm{gm} / \mathrm{cm}^{3}$, again based solely upon their composition. Values reported in the literature range from 1.19 to $1.24 \mathrm{gm} / \mathrm{cm}^{3}$ (Russell et al., 1980; Schalich et al., 1996). The lower value has been obtained by centrifugation in sucrose solutions in which, in our experience, flaviviruses are unstable. The higher values have been obtained by centrifugation in $\mathrm{CsCl}$. In any event, the lower density of packing of the lipids in the bilayer could result in a lower buoyant density than calculated. The lipids are assumed to have a density of 0.93 for these calculations, but their effective density in flaviviruses may be only half this, as described above, which would lead to a calculated density of 1.22 for the virion. Despite the uncertainties in the actual lipid composition of flaviviruses and uncertainties in the density of their packing, it is clear that a lipid bilayer centered at $R=163 \AA$ will accommodate the lipids of the virus.

\section{RNA Composition}

For rhinoviruses (Rossmann et al., 1985), the RNA occurs inside roughly a $106 \AA$ radius. The rhinovirus genome is about $7.8 \mathrm{~kb}$, and the flavivirus genome is about $10.7 \mathrm{~kb}$. Thus (making the worst possible assumption that the rhinovirus RNA completely fills the internal cavity, which it does not), the flavivirus RNA radius would have to be the cube root of 10.7/7.8 multiplied by $106 \AA$, giving an answer of $119 \AA$.

We state that the dengue virus capsid is situated between 105 and $135 \AA$ radius. But, as in many viruses, the basic sequence of the capsid protein (only $10 \mathrm{kDa}$ for dengue virus) would have to be associated with the RNA. Taking into account the low molecular weight of the capsid, it can easily be shown, were it all ordered, that it would only occupy a thickness of $15 \AA$ between 120 and $135 \AA$ radius. Hence, fully ordered RNA, while requiring a radius of only $118 \AA$, would be able to extend to $120 \AA$. In reality, the protein core is rather disordered and associated with RNA in an overlap region. Thus, there is plenty of room for the RNA genome.

\section{Interpretation of the CryoEM Map Using the Known Structure of the E Glycoprotein}

Trypsin treatment of TBEV yields a dimerized fragment that lacks the last 101 amino acids (Heinz et al., 1991) and whose X-ray crystallographic structure has been determined (Rey et al., 1995). The alphavirus glycoprotein E1 monomer was found to have a structure similar to 
the flavivirus E glycoprotein monomer (Lescar et al., 2001), but cryoEM reconstructions of alphaviruses demonstrated that E1 forms a different dimer interface than that observed in the TBEV E crystal structure (Lescar et al., 2001; Pletnev et al., 2001). Therefore, there was some concern whether the TBEV E dimer identified crystallographically would be present in mature dengue virus, although a TBEV subviral particle was interpreted in terms of the TBEV E crystallographic dimer (Ferlenghi et al., 2001).

In the absence of any guidance from quasisymmetry (Figures 1A and 3A), a quantitative technique was used for comparing different fits of the E glycoprotein structure into the dengue cryoEM map by using the EMfit program (Rossmann, 2000). In the first step, the TBEV E crystallographic dimer (Rey et al., 1995) was rotated about and translated around an icosahedral 2-fold axis to find the best fit. The procedure had to be performed four times because the absolute hand ("+" or "-") of the cryoEM map was unknown and because it was uncertain which end of the dimer axis was "up" or "down." The best fit was consistent with the preferred orientation suggested by Rey et al. (1995) ("down+" in Table 1A). As a control, the same procedure was applied using the E1 dimer arrangement found by fitting the TBEV E monomer to the Semliki Forest virus cryoEM map (Table 1B; Lescar et al., 2001; Pletnev et al., 2001). The resultant fits were far worse than those based upon the crystallographic TBEV E dimer structure. At this stage, a check was made of the magnification factor for the dengue cryoEM map using the down + structure (Table 1C). The best fit occurred when a pixel separation of 2.9 A was assumed, which is slightly larger than the nominal 2.8 A based upon the uncalibrated microscope magnification used to record the cryoEM images.

With an apparently reasonable fit for one monomer within an icosahedral asymmetric unit, it was then necessary to determine the number and orientation of additional monomers that could be modeled into the remaining, uninterpreted density. Thus, the number of pixels per icosahedral asymmetric unit that were occupied by the fitted monomer was compared with the number of unoccupied pixels of about the same height. Discounting the pixels of less than average height, it was found that there was space for another two monomers. Presumably, these monomers would form the same kind of dimer, since only dimers were released upon trypsin treatment of TBEV (Allison et al., 1995a). Furthermore, it would seem likely that the 2-fold axis of this second dimer would be oriented the same way (down) as the 2-fold axis of the first dimer. Inspection of the cryoEM map suggested that there might be a quasi-2-fold axis roughly between adjacent icosahedral 5 -fold and 3-fold axes. The densities at all pixels covered by the first fitted dimer were set to zero. The second dimer was then placed on a radial axis passing through a point near the quasi-2-fold axis. The rotation about, translation along, and orientation of the quasi-2-fold axis were refined with respect to the fit into the cryoEM map (Table 2). The procedure was carried out for both the down+ and down- results found when fitting the TBEV E dimer onto the icosahedral 2-fold axis (Table 1A). Only the down+ fit was viable because the down- fit generated a vast number of steric clashes around the icosahedral 3-fold axes between the dimers on the quasi-2-fold axes. The best down+ fit left few high-density pixels unoccupied and gave a faithful representation of the cryoEM dengue map (Figures 2A and 2B). 


\section{Acknowledgments}

We thank Sharon Wilder for help in preparation of the manuscript. The work was supported by a National Institutes of Health Program Project Grant to M.G.R., R.J.K., and T.S.B. (AI45976), grant AI20612 to J.H.S., grant AI10793 to J.H.S. and E.G.S., and a Purdue University redevelopment award. C.T.J. is a recipient of a National Institutes of Health biophysics training grant (GM08296).

\section{References}

Allison SL, Schalich J, Stiasny K, Mandl CW, Kunz C, Heinz FX. Oligomeric rearrangement of tickborne encephalitis virus envelope proteins induced by an acidic pH. J Virol. 1995a; 69:695-700. [PubMed: 7529335]

Allison SL, Stadler K, Mandl CW, Kunz C, Heinz FX. Synthesis and secretion of recombinant tickborne encephalitis virus protein $\mathrm{E}$ in soluble and particulate form. J Virol. 1995b; 69:5816-5820. [PubMed: 7637027]

Allison SL, Stiasny K, Stadler K, Mandl CW, Heinz FX. Mapping of functional elements in the stemanchor region of tick-borne encephalitis virus envelope protein. E J Virol. 1999; 73:5605-5612.

Allison SL, Schalich J, Stiasny K, Mandl CW, Heinz FX. Mutational evidence for an internal fusion peptide in flavivirus envelope protein. E J Virol. 2001; 75:4268-4275.

Baker TS, Olson NH, Fuller SD. Adding the third dimension to virus life cycles: three-dimensional reconstruction of icosahedral viruses from cryo-electron micrographs. Microbiol Mol Biol Rev. 1999; 63:862-922. [PubMed: 10585969]

Burke, DS.; Monath, TP. Flaviviruses. In: Knipe, DM.; Howley, PM., editors. In Fields Virology. 4th. Philadelphia: Lippincott Williams \& Wilkins; 2001. p. 1043-1125.

Caspar DLD, Klug A. Physical principles in the construction of regular viruses. Cold Spring Harb Symp Quant Biol. 1962; 27:1-24. [PubMed: 14019094]

Chambers TJ, Hahn CS, Galler R, Rice CM. Flavivirus genome organization, expression, and replication. Annu Rev Microbiol. 1990; 44:649-688. [PubMed: 2174669]

Chen Z, Stauffacher C, Li Y, Schmidt T, Bomu W, Kamer G, Shanks M, Lomonossoff G, Johnson JE. Protein-RNA interactions in an icosahedral virus at $3.0 \AA$ resolution. Science. $1989 ; 245: 154-159$. [PubMed: 2749253]

Cheng RH, Kuhn RJ, Olson NH, Rossmann MG, Choi HK, Smith TJ, Baker TS. Nucleocapsid and glycoprotein organization in an enveloped virus. Cell. 1995; 80:621-630. [PubMed: 7867069]

Corver J, Ortiz A, Allison SL, Schalich J, Heinz FX, Wilschut J. Membrane fusion activity of tickborne encephalitis virus and recombinant subviral particles in a liposomal model system. Virology. 2000; 269:37-46. [PubMed: 10725196]

Ferlenghi I, Clarke M, Ruttan T, Allison SL, Schalich J, Heinz FX, Harrison SC, Rey FA, Fuller SD. Molecular organization of a recombinant subviral particle from tick-borne encephalitis virus. Mol Cell. 2001; 7:593-602. [PubMed: 11463384]

Forsell K, Xing L, Kozlovska T, Cheng RH, Garoff H. Membrane proteins organizeasymmetrical virus. EMBO J. 2000; 19:5081-5091. [PubMed: 11013211]

Hahn YS, Galler R, Hunkapiller T, Dalrymple JM, Strauss JH, Strauss EG. Nucleotide sequence of dengue 2 RNA and comparison of the encoded proteins with those of other flaviviruses. Virology. 1988; 162:167-180. [PubMed: 2827375]

Heinz FX, Allison SL. Structures and mechanisms in flavivirus fusion. Adv Virus Res. 2000; 55:231, 269. [PubMed: 11050944]

Heinz FX, Mandl CW, Holzmann H, Kunz C, Harris BA, Rey F, Harrison SC. The flavivirus envelope protein E: isolation of a soluble form from tick-borne encephalitis virus and its crystallization. $\mathrm{J}$ Virol. 1991; 65:5579-5583. [PubMed: 1716695]

Heinz FX, Stiasny K, Püschner-Auer G, Holzmann H, Allison SL, Mandl CW, Kunz C. Structural changes and functional control of the tick-borne encephalitis virus glycoprotein $\mathrm{E}$ by the heterodimeric association with protein prM. Virology. 1994; 198:109-117. [PubMed: 8259646]

Kielian M. Membrane fusion and the alphavirus life cycle. Adv Virus Res. 1995; 45:113-151. [PubMed: 7793323] 
Kielian M, Klimjack MR, Ghosh S, Duffus WA. Mechanisms of mutations inhibiting fusion and infection by Semliki Forest virus. J Cell Biol. 1996; 134:863-872. [PubMed: 8769412]

Larson SB, Koszelak S, Day J, Greenwood A, Dodds JA, McPherson A. Three-dimensional structure of satellite tobacco mosaic virus at $2.9 \AA$ resolution. J Mol Biol. 1993; 231:375-391. [PubMed: 8510153]

Lee S, Owen KE, Choi HK, Lee H, Lu G, Wengler G, Brown DT, Rossmann MG, Kuhn RJ. Identification of a protein binding site on the surface of the alphavirus nucleocapsid and its implication in virus assembly. Structure. 1996; 4:531, 541. [PubMed: 8736552]

Lenard, J. Lipids of alphaviruses. In: Schlesinger, RW., editor. The Togaviruses. New York: Academic Press; 1980. p. 335-341.

Lescar J, Roussel A, Wein MW, Navaza J, Fuller SD, Wengler G, Wengler G, Rey FA. The fusion glycoprotein shell of Semliki Forest virus: an icosahedral assembly primed for fusogenic activation at endosomal pH. Cell. 2001; 105:137-148. [PubMed: 11301009]

Lindenbach, BD.; Rice, CM. Flaviviridae: the viruses and their replication. In: Knipe, DM.; Howley, PM., editors. Fields Virology. 4th. Philadelphia: Lippincott Williams \& Wilkins; 2001. p. 991-1041.

Mandl CW, Guirakhoo F, Holzmann H, Heinz FX, Kunz C. Antigenic structure of the flavivirus envelope protein $\mathrm{E}$ at the molecular level, using tick-borne encephalitis virus as a model. J Virol. 1989; 63:564-571. [PubMed: 2463377]

Mandl CW, Kroschewski H, Allison SL, Kofler R, Holzmann H, Meixner T, Heinz FX. Adaptation of tick-borne encephalitis virus to BHK-21 cells results in the formation of multiple heparan sulfate binding sites in the envelope protein and attenuation in vivo. J Virol. 2001; 75:5627-5637. [PubMed: 11356970]

Paredes AM, Brown DT, Rothnagel R, Chiu W, Schoepp RJ, Johnston RE, Prasad BVV. Threedimensional structure of a membrane-containing virus. Proc Natl Acad Sci USA. 1993; 90:90959099. [PubMed: 8415660]

Pletnev SV, Zhang W, Mukhopadhyay S, Fisher BR, Hernandez R, Brown DT, Baker TS, Rossmann MG, Kuhn RJ. Locations of carbohydrate sites on Sindbis virus glycoproteins show that E1 forms an icosahedral scaffold. Cell. 2001; 105:127, 136. [PubMed: 11301008]

Rey FA, Heinz FX, Mandl C, Kunz C, Harrison SC. The envelope glycoprotein from tick-borne encephalitis virus at $2 \AA$ resolution. Nature. 1995; 375:291-298. [PubMed: 7753193]

Roehrig JR, Bolin RA, Kelly RG. Monoclonal antibody mapping of the envelope glycoprotein of the dengue 2 virus Jamaica. Virology. 1998; 246:317-328. [PubMed: 9657950]

Rossmann MG. Fitting atomic models into electron microscopy maps. Acta Crystallogr D. 2000; 56:1341-1349. [PubMed: 10998631]

Rossmann MG, Arnold E, Erickson JW, Frankenberger EA, Griffith JP, Hecht HJ, Johnson JE, Kamer G, Luo M, Mosser AG, et al. Structure of a human common cold virus and functional relationship to other picornaviruses. Nature. 1985; 317:145-153. [PubMed: 2993920]

Russell, PK.; Brandt, WE.; Dalrymple, JM. Chemical and antigenic structure of flaviviruses. In: Schlesinger, RW., editor. The Togaviruses Biology Structure Replication. New York: Academic Press; 1980. p. 503-529.

Schalich J, Allison SL, Stiasny K, Mandl CW, Kunz C, Heinz FX. Recombinant subviral particles from tick-borne encephalitis virus are fusogenic and provide a model system for studying flavivirus envelope glycoprotein functions. J Virol. 1996; 70:4549-4557. [PubMed: 8676481]

Schulz GE. $\beta$-barrel membrane proteins. Curr Opin Struct Biol. 2000; 10:443-447. [PubMed: 10981633]

Skehel JJ, Wiley DC. Coiled coils in both intracellular vesicle and viral membrane fusion. Cell. 1998; 95:871-874. [PubMed: 9875840]

Skoging U, Vihinen M, Nilsson L, Liljeström P. Aromatic interactions define the binding of the alphavirus spike to its nucleocapsid. Structure. 1996; 4:519-529. [PubMed: 8736551]

Strauss JH, Strauss EG. The alphaviruses: gene expression, replication, and evolution. Microbiol Rev. 1994; 58:491-562. [PubMed: 7968923]

Strauss JH, Strauss EG. Virus evolution: how does an enveloped virus make a regular structure? Cell. 2001; 105:5-8. [PubMed: 11300997] 
Subramaniam S, Henderson R. Molecular mechanism of vectorial proton translocation by bacteriorhodopsin. Nature. 2000; 406:653-657. [PubMed: 10949309]

Vashishtha M, Phalen T, Marquardt MT, Ryu JS, Ng AC, Kielian M. A single point mutation controls the cholesterol dependence of Semliki Forest virus entry and exit. J Cell Biol. 1998; 140:91-99. [PubMed: 9425157]

World Health Organization. Dengue and dengue haemorrhagic fever. Fact sheet. 1998; (117) 


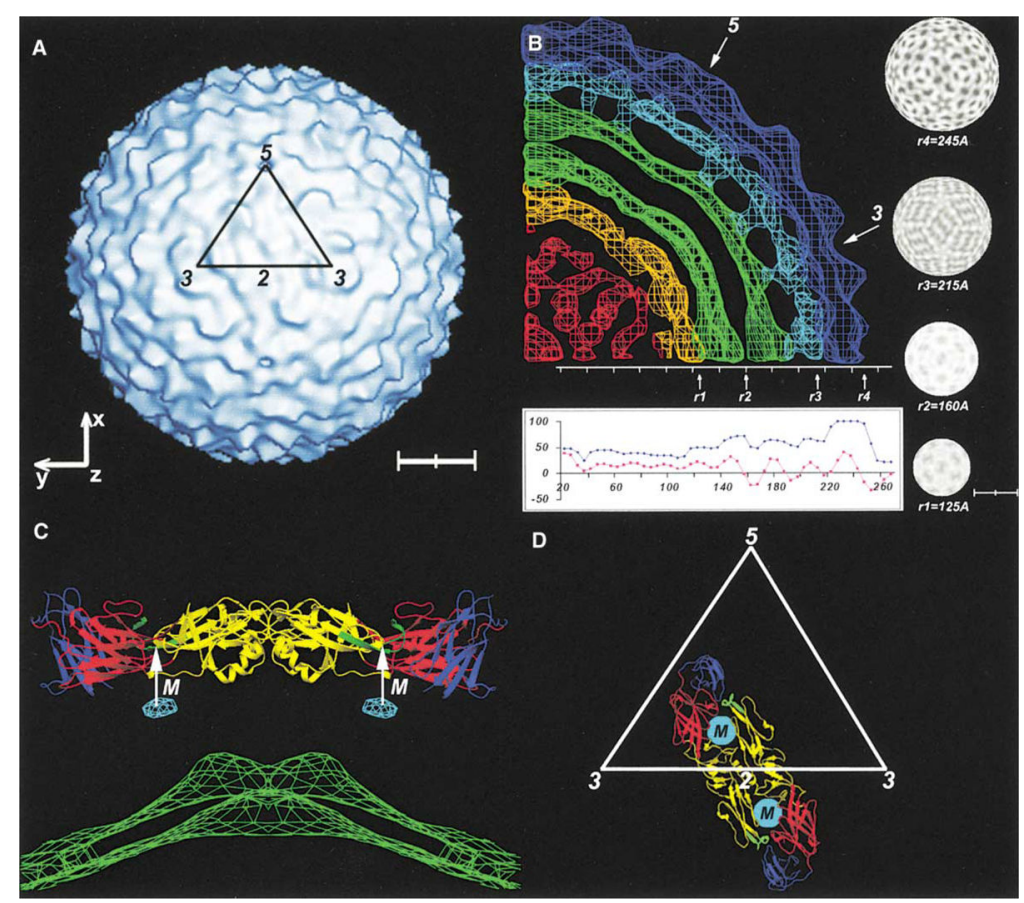

Figure 1. The CryoEM Density

(A) Surface-shaded representation of dengue- 2 cryoEM reconstruction at $24 \AA$ resolution, showing the outline of one icosahedral asymmetric unit and the definition of the coordinate system. Scale bar represents $100 \AA$.

(B) Central crosssection showing the cryo-EM density with a plot of the maximum (blue) and averaged (purple) density. Arrows indicate the position of the 5-fold and 3 -fold axes. Shown also are radial density sections at the defined radii, r1, r2, r3, and r4. Higher density representing protein is shown in dark shading. Scale bar represents $175 \AA$ A.

(C) Ribbon drawing of the E dimer situated on an icosahedral 2-fold axis, showing the largest uninterpreted electron density peak outside the lipid bilayer, probably representing the $\mathrm{M}$ protein (light blue), located close to the hole between the E dimers. The white arrow indicates the position of the dimer holes. The outer leaflet of the lipid bilayer is shown in green. The domains I, II, and III of an E monomer are shown in red, yellow, and blue, respectively. The fusion peptides are in green.

(D) Ribbon drawing showing the position and orientation of the E dimer associated with an icosahedral 2-fold axis. Shown in white is the outline of one icosahedral asymmetric unit. The domains of $\mathrm{E}$ are colored as in (C). The portion of the membrane protein $\mathrm{M}$ below the dimer is indicated. 

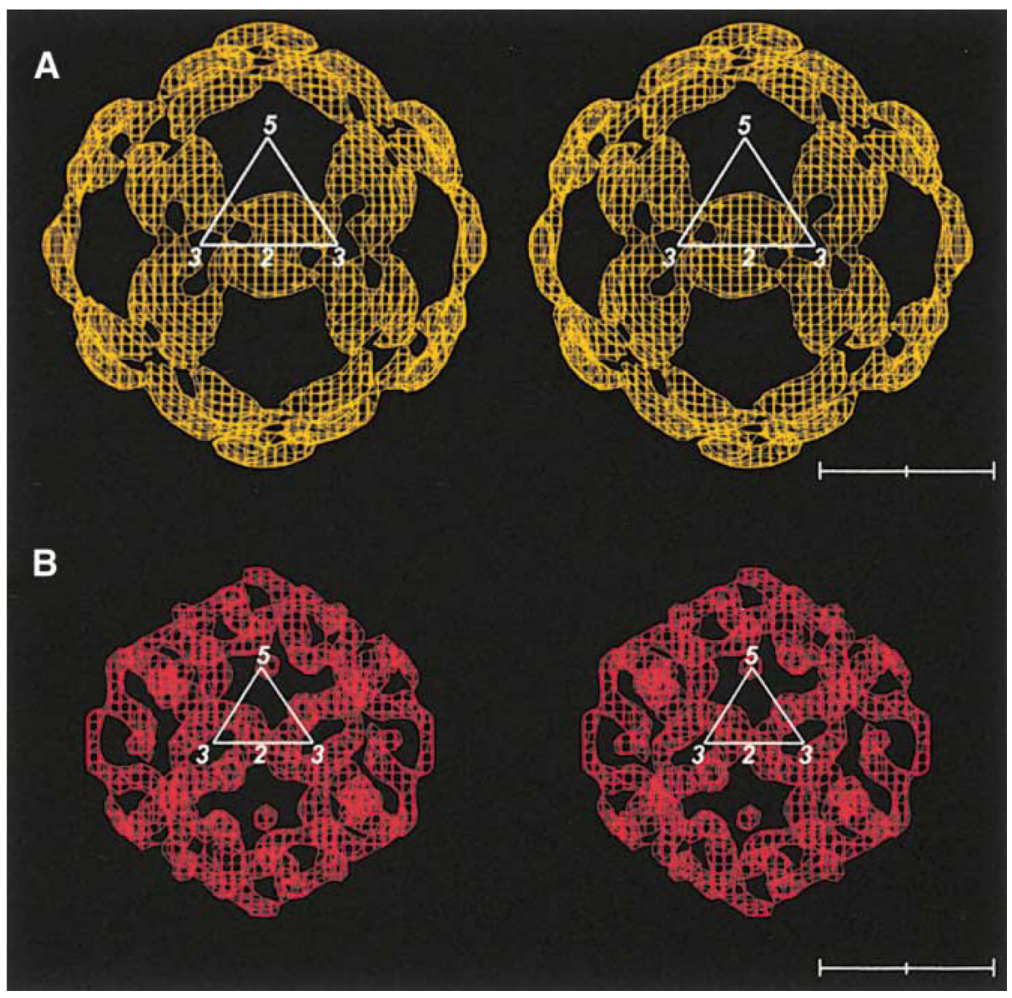

Figure 2. CryoEM Density of the Nucleocapsid Shell and the RNA

(A) Stereo diagram of the region corresponding to the yellow nucleocapsid shell in Figure 1, between 105 and $135 \AA$ radii.

(B) Stereo diagram of density corresponding to the RNA region of the core (red in Figure 1), inside a radius of $105 \AA$. Only one hemisphere is shown. Scale bars represent $100 \AA$. 

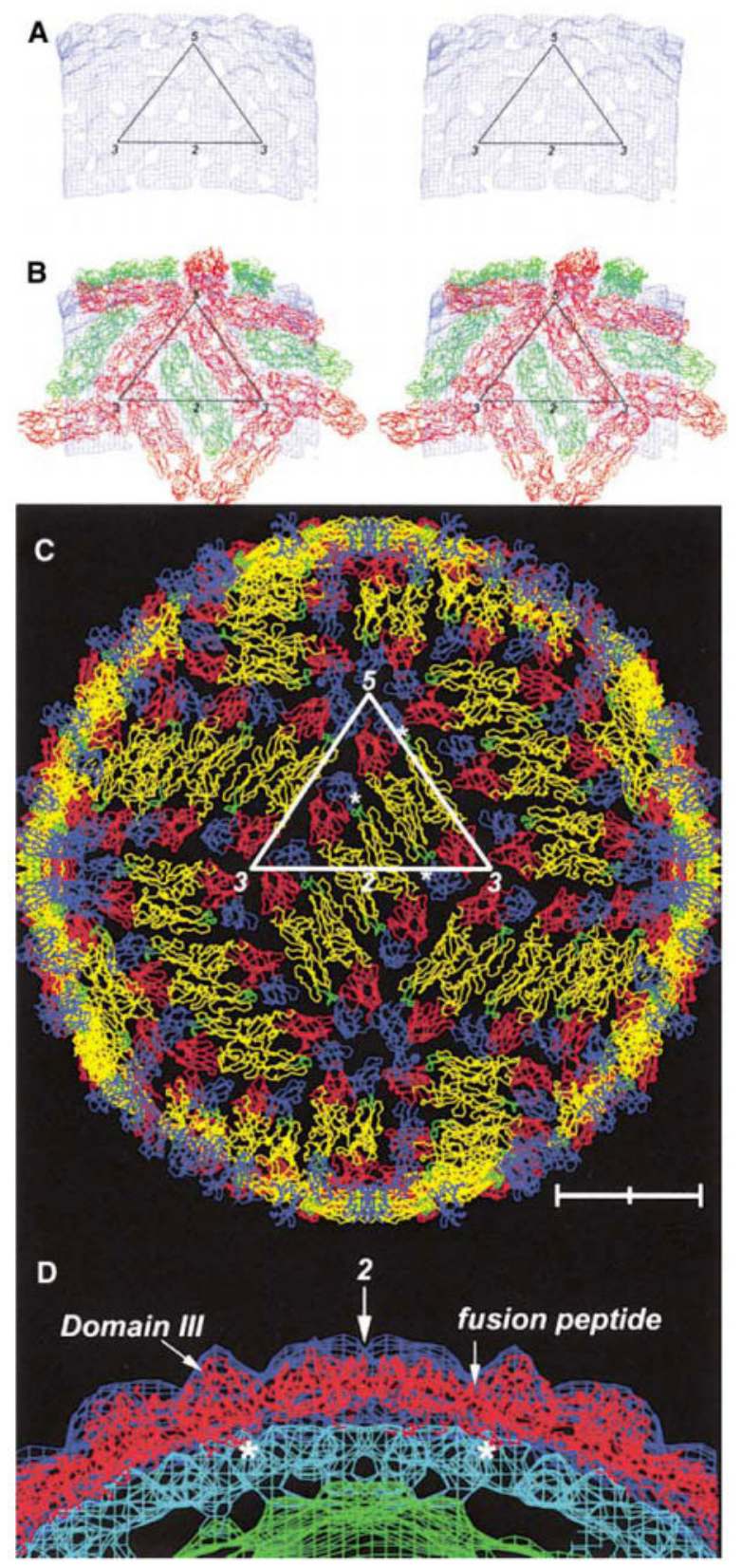

Figure 3. Fit of E Dimers into Density

(A) and (B) correspond to density that is between planes, perpendicular to an icosahedral 2fold axis and at a distance of 220-250 A from the viral center. The contour level is at about $4 \sigma$ (root mean square deviation from the mean density).

(A) Stereo diagram showing only the density between 220 and 245 A radius.

(B) Stereo diagram showing the interpreted density in terms of dimers on icosahedral 2-fold axes (green) and dimers on quasi-2-fold axes (red).

(C) Structure of the whole virus showing each monomer with domains I, II, and III in red, yellow, and blue, respectively. The fusion peptide is shown in green. The C-terminal residue 
395 is shown as a white asterisk for monomers within the defined icosahedral asymmetric unit. Note the pair of holes in each dimer. Scale bar represents $100 \mathrm{~A}$.

(D) Central crosssection through the cryo-EM density showing the outer radial shells as in Figure 1B. The direction of the crosssection follows the length of a dimer situated on an icosahedral 2-fold axis. The arrow indicates the position of the 2-fold axis. The location of domain III and the fusion peptide in domain II are shown. Asterisks indicate the carboxy end of the fitted E protein. 


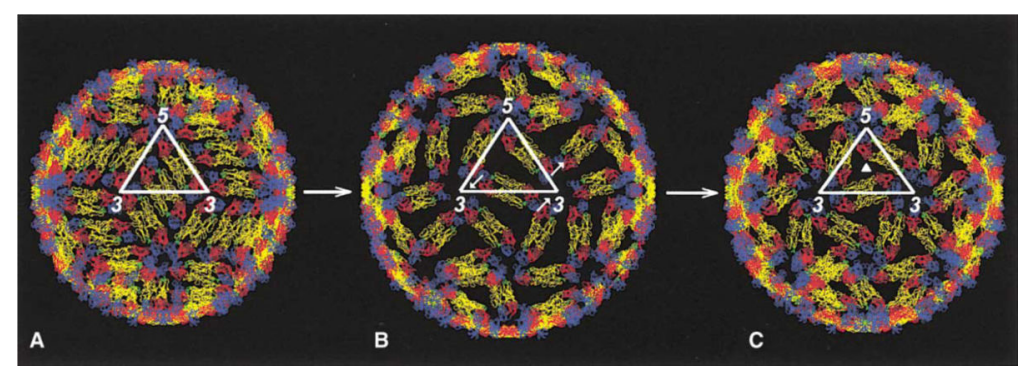

Figure 4. Proposed Rearrangement of $\mathbf{E}$ Dimers in Flaviviruses upon Exposure to Low pH The E protein dimers in mature virus (A) are shown undergoing a rearrangement to the predicted $T=3$ fusogenic structure (C) with a possible intermediate (B). Arrows in (B) indicate the direction of $\mathrm{E}$ rotation. The solid triangle in $(\mathrm{C})$ indicates the position of a quasi-3-fold axis. Note the radial expansion of the particle in (B) and (C). 


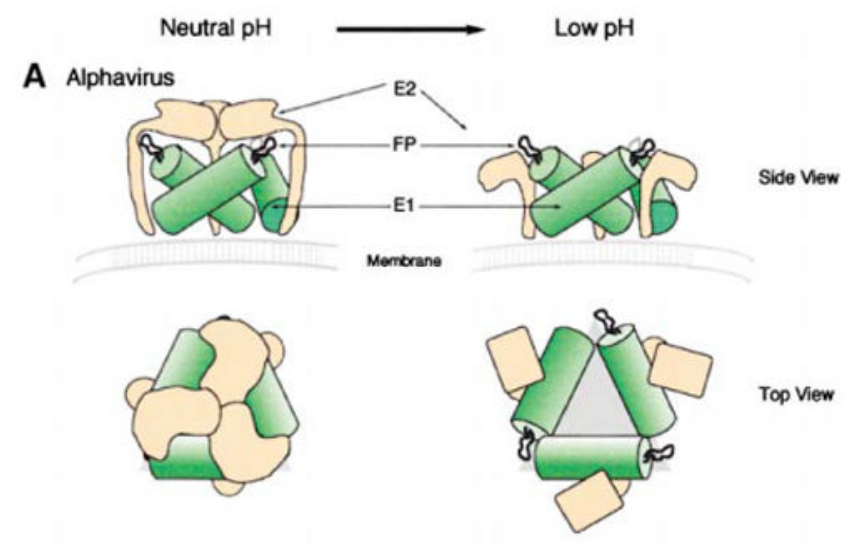

B Flavivirus

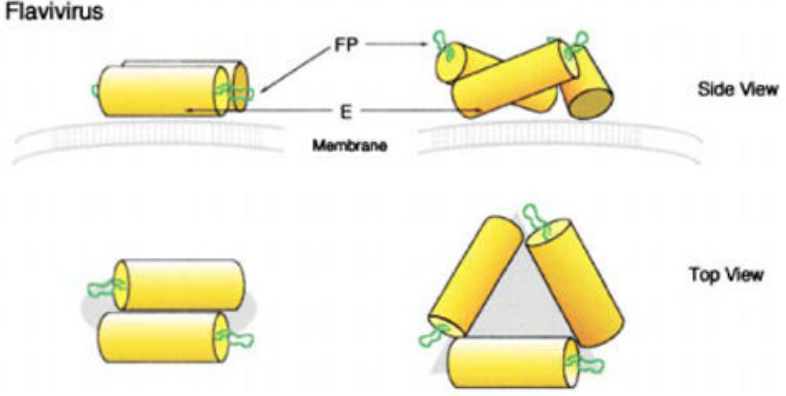

Figure 5. Configuration of Glycoproteins of Alphaviruses and Flaviviruses on the Surface of Virions at Neutral pH and the Proposed Configuration at Acid pH

(A) In the alphaviruses, E1 glycoproteins are shown as green cylinders, E2 glycoproteins as tan shapes, and the fusion peptide (FP) as a black curved line.

(B) In flaviviruses, E glycoproteins are shown as yellow cylinders with the fusion peptide as a green curve. In both (A) and (B), the membrane is shown in gray. 
든

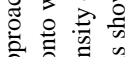

을

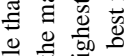

可.

입 욤

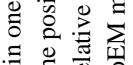

클

产资

t) $\overrightarrow{3} \overline{0}$

离总焉

D.

暂

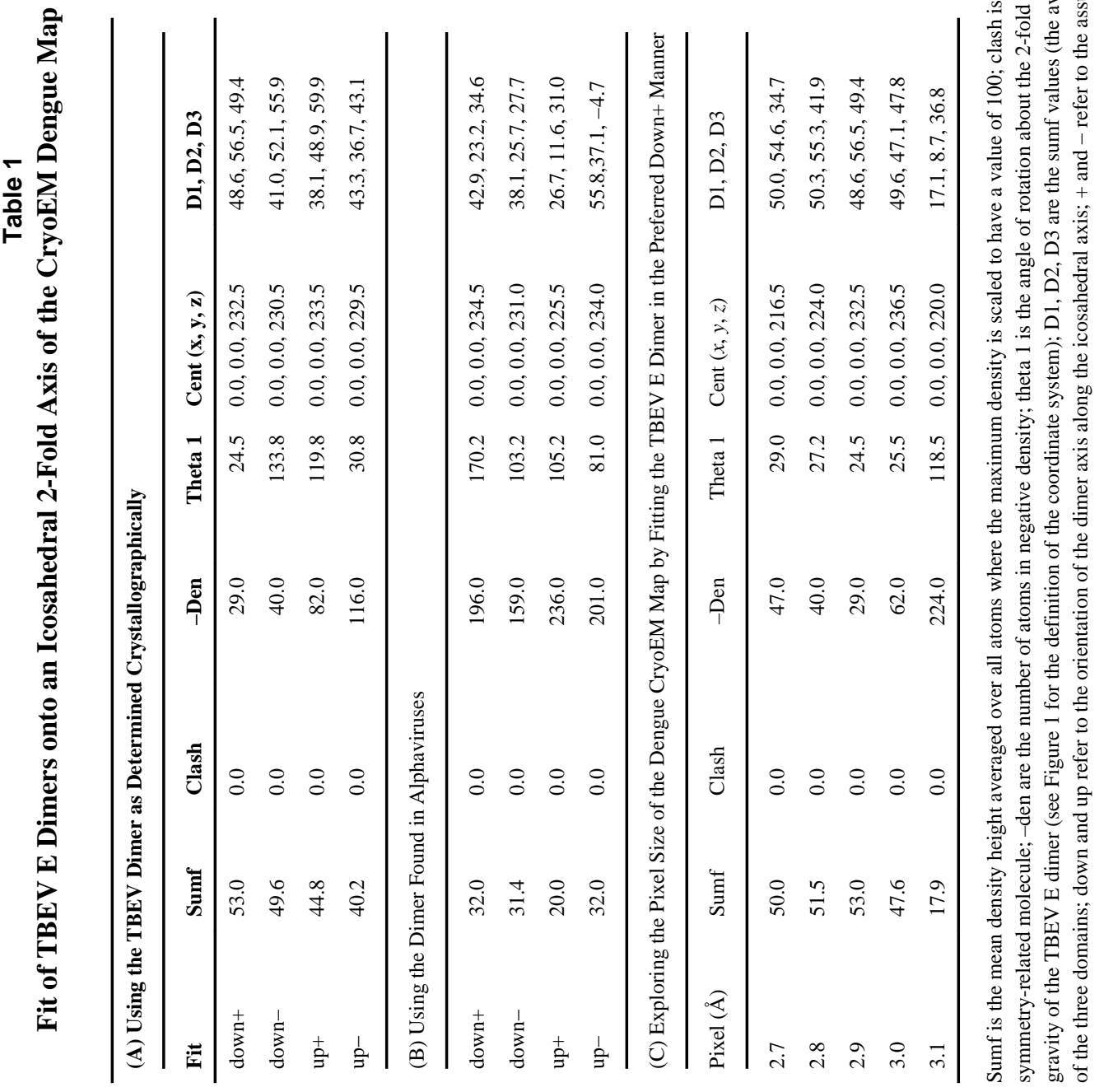




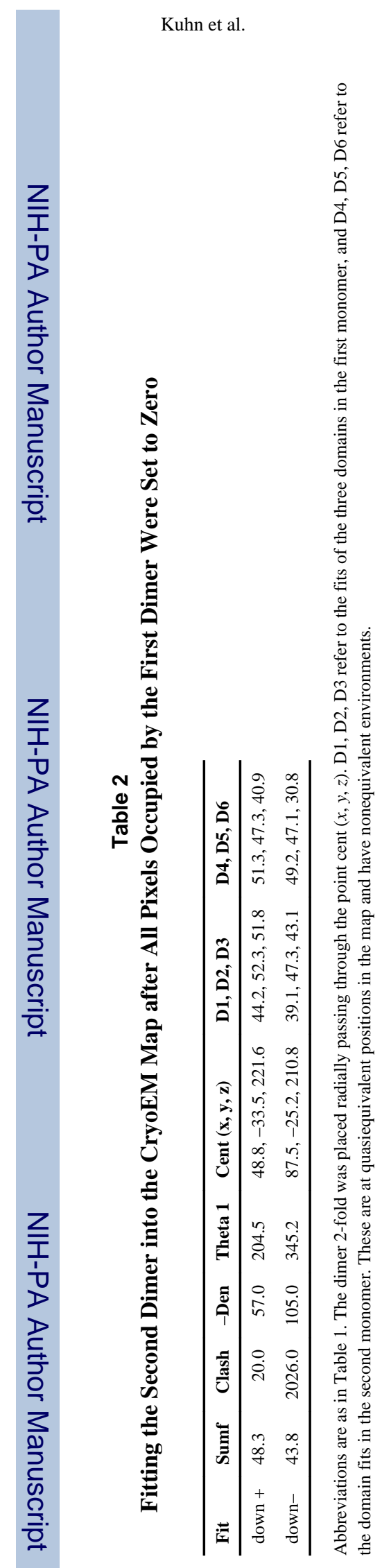

Cell. Author manuscript; available in PMC 2014 September 03. 